\title{
IR GEMINORUM: COMPLEX DISK BEHAVIOR DURING QUIESCENCE
}

\author{
Zongyun Li, ${ }^{1}$ Kam-Ching Leung, ${ }^{2}$ and C. Martin Gaskell ${ }^{2}$ \\ RESUMEN
}

Hemos realizado fotometría de IR Gem en la banda V casi continuamente durante más de 6 días, desde los observatorios de Yunnan (China) y Behlen (Nebraska, EU), comenzando tres días después de una erupción normal en enero del 2002. Nuestras observaciones muestran que el comportamiento de esta estrella de tipo SU UMa es sorprendentemente complicado, y que para IR Gem la etapa quiescente es potencialmente más interesante que las erupciones. Encontramos una modulación fotométrica con un período de $98.5 \mathrm{~min}$, exactamente igual al período orbital determinado espectroscópicamente. Tentativamente, atribuímos ésto al calentamiento de la secundaria. Durante los primeros tres días apareció una modulación con un período $5 \%$ mayor que el período orbital. Sugerimos que ésto puede ser señal de la precesión apsidal de un disco excéntrico. A la mitad de nuestras observaciones apareció una modulación con un período $3 \%$ menor que el orbital. Para su explicación, hacemos uso de la precesión del nodo. Encontramos otra modulación más lenta, con un período de unos 1.7 días, el cual es congruente con el período esperado para la precesión del nodo. Encontramos también una extraña modulación con un período de $4.3 \mathrm{~d}$, que sospechamos pueda ser resultado de la resonancia entre las frecuencias de la precesión nodal y la apsidal. También observamos ciclos cuasi-periódicos inexplicables, con escalas de tiempo que varían entre $\sim 0.2 \mathrm{y} \sim 0.4$ días.

\section{ABSTRACT}

We have carried out nearly continuous V-band photometry from Yunnan Observatory (China) and Behlen Observatory (Nebraska, USA) of IR Gem for over six days starting three days after a normal outburst in January 2002. Our observations show that the behavior of this SU UMa star is unexpectedly complicated, and that for IR Gem, quiescence is potentially more interesting than outbursts. We find a photometric modulation with a period of $98.5 \mathrm{~min}$, exactly equal to the spectroscopically determined orbital period. We tentatively attribute this to heating of the secondary. During the first three days a modulation appeared with a period $5 \%$ longer than the orbital period. We suggest that this might be a signature of apsidal precession of an eccentric disk. During the middle of our period of observations a modulation with a period $3 \%$ shorter than the orbital period appeared. We invoke nodal precession to explain this. A slower modulation we found with a period of about $1.7 \mathrm{~d}$ is roughly consistent with the expected period of nodal precession. There is a puzzling $4.3 \mathrm{~d}$ period modulation that we suspect may be the result of beating between apsidal and nodal precession frequencies. We also find inexplicable quasi-periodic cycles on timescales drifting from $\sim 0.2$ to $\sim 0.4$ days.

\section{Key Words: ACCRETION, ACCRETION DISKS - BINARIES: PHOTOMETRIC - NOVAE, CATA- CLYSMIC VARIABLES - STARS: INDIVIDUAL (IR GEMINORUM)}

\section{INTRODUCTION}

IR Gem is a member of the SU UMa subclass of dwarf novae. SU UMa stars are identified by their distinctive superoutbursts and the accompanying superhumps phenomena (see Warner $1995 \S 3.6$ for a comprehensive review). Szkody, Shafter \& Cowley (1984, hereafter SSC) discovered IR Gem to be an SU UMa star and carried out the first systematic investigation. They determined a characteristic superhump period of $102 \mathrm{~min}$ from photometric data collected during a superoutburst, and detected a 101 min modulation (with amplitude 0.4-0.6 mag in the

\footnotetext{
${ }^{1}$ Nanjing University, China.

${ }^{2}$ University of Nebraska, USA.
}

$V$ band) at quiescence, which was interpreted to be an orbital effect of irradiation of the secondary. Further, based on the small $\mathrm{K} 1$ value $(30 \pm 4 \mathrm{~km} / \mathrm{s})$ and the strong "orbital" modulation, the authors presumed that IR Gem consisted of a massive white dwarf and possibly a heated degenerate secondary. However, the paper left a confusing problem that the "orbital" modulation of $101 \mathrm{~min}$ is slightly but not negligibly discrepant with the 98.5 min periodicity obtained from radial velocity curve of the $H_{\alpha}$ line.

Lázaro et al. (1991) have confirmed the 98.5 min spectroscopic period in phase-dependent $\mathrm{EW}$ variations of $H_{\alpha}$ and Woods et al. (1992) have confirmed 
it in the UV resonance lines. It has also been found in the radial velocity curve of $H_{\beta}$ and $H_{\gamma}$ (Feinswog, Szkody \& Garnavich 1988). Nevertheless, the photometric modulation in the quiescent state behaved erratically: sometimes it seems to have almost disappeared (Feinswog et al. 1988), but then it regained a larger amplitude again (Szkody et al. 1989). Szkody et al. (1989) suggested that the modulation amplitude might depend on outburst phase since the former light curve was obtained just before an outburst while the latter was 8 days after an outburst (the average outburst period of IR Gem is 21 days. Note also, the 101 min modulation SSC described was observed 5 days before an outburst). However, previous photometry was not adequate to address this issue. In this paper we report time-resolved photometry of IR Gem starting 3 days after an outburst maximum and lasting over 6 days. Our observations trace the changing course of the "orbital" modulation and are thus helpful for understanding its erratic behavior.

\section{OBSERVATIONS}

We made almost continuous CCD V-band photometric observations of IR Gem in quiescence for a period of 6 days (from 2002 January 19 to 2002 January 25, UT) at Yunnan Observatory (YNO) in China and the University of Nebraska's Behlen Observatory (BO) in the USA. The time difference between the two sites is approximately eleven hours. A total of 1783 useful frames were processed as described in Gao et al. (1999). A full log and further details of our observations and analyses can be found in Fu et al. (2004).

Differential magnitudes of IR Gem were obtained using 2 secondary photometric standards, stars 5 and 6 on the finding chart of Misselt (1996), as the comparison star (C) and the check star (K), respectively. $¿$ From the method suggested by Howell, Warnock \& Mitchell (1988), we estimate the standard deviation of the differential magnitudes of $\mathrm{V}-\mathrm{C}$ at $0.02-0.03$ mag for YNO data and 0.03-0.04 mag for Behlen data.

\section{RESULTS AND ANALYSIS}

IR Gem had reached the maximum of a normal outburst just three days before the start of our observations (Mattei 2002). All of our photometric data are shown in Figure 1. Since the data were collected in two hemispheres, the observations mostly lasted 16-21 hours (with a gap of 1 or 2 hours) every day and so provide a good database for period analysis. Fig. 1 also shows the best linear fit to the light curve. From this fit we estimate that the rate of decline was $0.135(3) \mathrm{mag} / \mathrm{d}$.

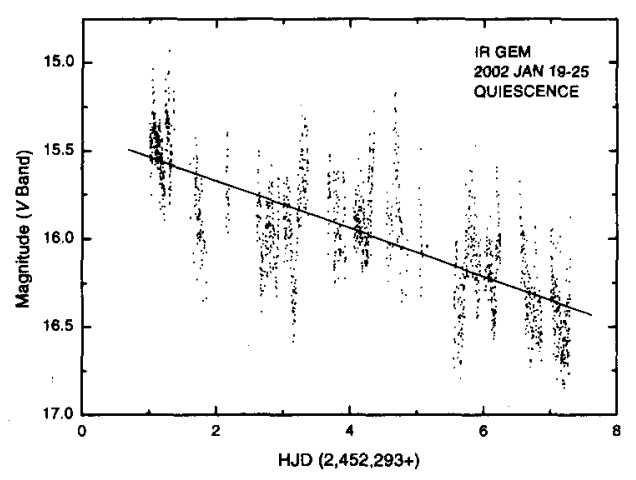

Fig. 1. Full light curve of the observations. The ordinate presents the estimated magnitudes based on a comparison star $V$ magnitude of $13.761(30)$. The solid line shows the best linear fit to the data.

\subsection{Signals Near The Orbital Frequency}

In the complicated power spectrum of the star (see Fig. 3), there are only small spikes at $14.62(2)$ and $15.17(2) \mathrm{c} / \mathrm{d}$ around the orbital frequency. In order to study the modulations, one should remove the longer-period components. In our case, we smoothed the light curve using the method of a sliding Gaussian window with $\sigma$ of $0.05 \mathrm{~d}$.

We then subtracted the smoothed curve to produce residuals. The smoothing interval was chosen to provide the most effective suppression of the lower-frequency components and the highest signalto-noise ratio in the vicinity of the orbital frequency.

In the CLEAN spectrum (Roberts, Lehar \& Dreher 1987) of the residuals, a prominent peak became visible at a frequency of $14.61(2) \mathrm{c} / \mathrm{d}$, i.e., a period of $98.5613 \mathrm{~min}$, which exactly equals the spectroscopically-determined orbital period (Szkody et al. 1984; Feinswog et al. 1988). The folded light curve at this frequency shows a sinusoidal profile with a peak-to-trough amplitude of $\sim 0.08$ mag.

Our good temporal coverage enables us to do period analyses on 2-day long segments of the residuals separately. The results are presented in Fig. 2. Only one peak at $13.90(5) \mathrm{c} / \mathrm{d}$ occurs between 10 and 16 c/d in the CLEAN spectrum of Part I (runs 1 to 4); the most prominent feature appears at $15.10(6) \mathrm{c} / \mathrm{d}$ for Part II (runs 5 to 8); and for Part III (the last 5 runs), a remarkable peak occurs at $14.58(6) \mathrm{c} / \mathrm{d}$. Note that the periods 103.6(4) and 95.4(4) min, corresponding to the frequencies 13.90 and $15.10 \mathrm{c} / \mathrm{d}$, are $5.2 \%$ longer and $3.1 \%$ shorter than the orbital period of $98.5 \mathrm{~min}$, respectively. Also shown in Fig. 2 are waveforms of the segments folded at frequen- 


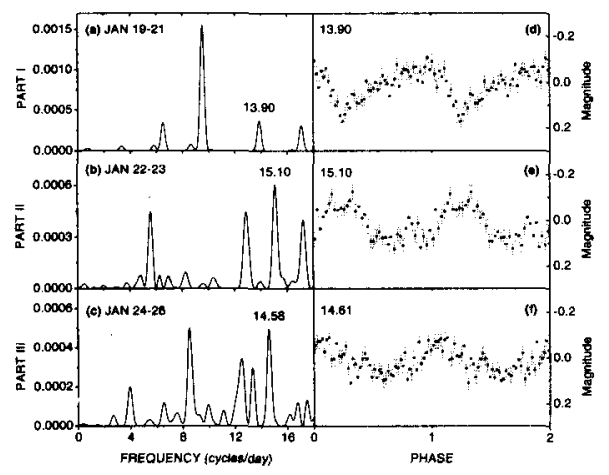

Fig. 2. Period analysis of separate 2-day long segments of the residuals after subtraction of lower frequency components. $(a)(b)(c)$, CLEAN spectra of each segment. $d)$ $(e)(f)$, Wave forms of each segment. Folding frequencies are labelled on the left of each frame.

cies $13.90,15.10$ and $14.61 \mathrm{c} / \mathrm{d}$, respectively. Monte Carlo simulations show that the peaks are $98 \%$ significant.

\subsection{Variation on a 4-5 Day Timescale}

We computed the CLEAN spectrum of the complete set of de-trended data (Fig. 3) which had been generated by subtracting the linear decline from the original light curve. The highest peak in the spectrum appears at the frequency of $0.21(3) \mathrm{c} / \mathrm{d}$. Although the time series is short for determining a period of 4-5 d, the sinusoidal wave with a period of $\sim 4.3 \mathrm{~d}$ and a full amplitude of $\sim 0.24 \mathrm{mag}$ is quite evident in the raw light curve (Fig. 1). This strong modulation has not been found before, since previous photometry lasted at most three days in quiescence.

\subsection{Apparent Quasi-Periodic Cycles}

In the light curve, there are prominent largeamplitude (0.15-0.6 mag) cycles that occurred in a quasi-periodic fashion. A similar cycle with the amplitude of $0.5 \mathrm{mag}$ can also be seen in the light curve of Feinswog et al. (1988). On the other hand, the second highest peak stands at frequency of 3.66(2) $\mathrm{c} / \mathrm{d}$ in Fig. 3 with the lower peaks in its vicinity. After subtracting the variation at $0.21 \mathrm{c} / \mathrm{d}$, the residuals were folded at $3.66(2) \mathrm{c} / \mathrm{d}$ and show a sinusoidal waveform with full amplitude of $0.19(8)$ mag. The mechanism underlying this modulation is not clear.

\section{DISCUSSION}

We believe the modulation at $14.62 \mathrm{c} / \mathrm{d}$ is certainly an orbital effect. It has an amplitude of $\sim 0.08$ mag and a period exactly equal to that deduced from

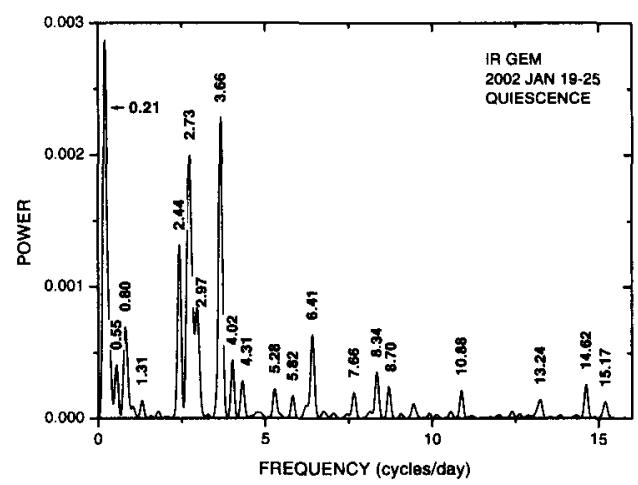

Fig. 3. CLEAN spectrum of the complete set of the detrended time series, showing a strong signal at $0.21 \mathrm{c} / \mathrm{d}$. The complicated pattern suggests multi-periods. Spikes at 14.62 and $15.17 \mathrm{c} / \mathrm{d}$ can also be seen.

spectroscopy. Unfortunately, the ephemeris available is not accurate enough to check at what phase the modulation is a maximum, and thus we can not tell definitely whether it is due to the hot spot or just heating of the secondary. We tentatively attribute the modulation to the latter because of its sinusoidal waveform. Because it has a small amplitude, this modulation does not suffer from the energetics problem posed by SSC where the 0.5 mag modulation observed in quiescence needs an X-ray flux 10 times higher than the observed level if it is a heating effect.

We suggest that the mechanism underlying the $13.90 \mathrm{c} / \mathrm{d}$ modulation appearing in the first three days, is similar to that of a late superhump (Hessman et al.,1992). Its light source should mainly be the hot spot, where the inflow stream strikes the disk, because the mean profile of the modulation (Fig.2 (d)) shows a slow rise followed by a steep decline. This is a typical feature of hot spot modulations. The longer period $0.21 \mathrm{c} / \mathrm{d}$ variation could easily be explained as beating between the orbital motion and the apsidal precession of an eccentric disk. It is reminiscent of the 0.5 mag 101 min modulation caught by SSC. We suspect that it is also produced by the hot spot rather than irradiation of the secondary. The larger amplitude simply indicates a larger $\dot{M} 2$.

In our second segment, a sinusoidal modulation with a period of $\sim 95.4$ min dominates the power spectrum. This could be explained by an episodically tilting disk (Harvey et al, 1998). When disk tilting occurs, the mass transfer stream overshoots its rim, and thus we could no longer see the hot spot modulation. The disk's node immediately begins retrograde precession in the orbital plane, and 
the negative superhump (the 95-min modulation) is thus produced.

According to the nodal precession model, the negative superhump period of $95.4 \mathrm{~min}$ and the orbital period of 98.5 min demand that the tilted disk be retrogradely precessing at a rate of $2.08(35)$ degrees per cycle. We do indeed find that a spike appears at $\sim 0.55 \mathrm{c} / \mathrm{d}$ in the CLEAN spectrum for the complete set (Fig. 3), while a higher peak at $\sim 0.6$ c/d appears for the runs 4 to 13 (not shown). These features provide independent evidence of the validity of the model.

If apsidal and nodal precessions function simultaneously in the disk of IR Gem, the modulation at $0.21(3) \mathrm{c} / \mathrm{d}$ in Fig. 3 or the $\sim 4.3 \mathrm{~d}$ sinusoidal variations visible in Fig. 1 can be tentatively understood as beating between the two modes. We suspect that the spikes at $\sim 0.55$ and $\sim 0.8 \mathrm{c} / \mathrm{d}$ might be the signatures of precession of the disk. Correlations between the variations in intensities of orbital modulation, positive and negative superhumps, and phase of the $4.3 \mathrm{~d}$ cycle could support this suggestion to some extent. Unfortunately, our observations are not adequate for us to address this problem. More extensive observations are needed.

Although it is rare to observe superhumps in quiescent states, there have been other well-documented examples. AL Com showed persistent superhumps as well as orbital humps at quiescence (Patterson et al. 1996). EG Cnc sustained its late superhumps for at least $90 \mathrm{~d}$ after the 1996 superoutburst (Patterson et al. 1998). ER UMa, which has an extremely short superoutburst cycle and essentially no quiescence, also showed superhumps in the minima of outbursts (Gao et al. 1999). More recently, Woudt and Warner (2001) found V359 Cen showing superhumps with a period of $112 \mathrm{~min}$ and V630 Sgr to be an eclipsing permanent superhumper with evidence of disk precession in both the complete set of raw light curves and in the Fourier amplitude spectrum.

In conclusion our observations show that the behavior of IR Gem in quiescence is unexpectedly complicated and that further photometric study at qui- escence will be invaluable. Additional spectroscopy to improve the ephemeris would also be valuable. Clearly for IR Gem quiescence is as interesting as outbursts.

This research was supported in part by grants 10173005 and 10010120074 from the National Natural Science Foundation of the People's Republic of China. We wish to thank Hai Fu, Zili $\mathrm{Li}$, and Zhouseng Zhang for their assistance in the observations and analysis.

\section{REFERENCES}

Feinswog, L., Szkody, P., \& Garnavich, P. 1988, AJ, 96, 1702

Fu, H., Li, Z.-Y., Leung, K.-C., Zhang, Z.-S., Li, Z.-L., \& Gaskell, C. M. 2004, ChJAA, 4,88

Gao, W. H., Li, Z. Y., Wu, X. A., Zhang, Z. S., \& Li, Y. 1999, ApJ, 527, L55

Harvey, D. A., Skillman, D. R., Kemp, J., Patterson, J., Vanmunster, T., Fried, R. E., \& Retter, A. 1998, ApJ, 493, L105

Hessman, F. V., Mantel, K.-H., Barwig, H., \& Schoembs, R. 1992, A\&Ap, 263, 147

Howell, S. B., Warnock III, A., \& Mitchell, K. J. 1988, AJ, 95, 247

Lázaro, C., Martínez-Pais, I. G., Arévalo, M. J. \& Solheim, J. E. 1991, AJ, 101, 196

Mattei, J. A. 2002, Observations from the AAVSO International Database, private communication.

Misselt, K. A. 1996, PASP, 108, 146

Patterson, J., Augusteijn, T., Harvey, D. A., Skillman, D. A., Abbott, T. M. A., \& Thorstensen, J. 1996, PASP, 108, 748

Patterson, J., et al. 1998, PASP, 110, 1290

Roberts, D. H., Lehar, J., \& Dreher, J. W. 1987, AJ, 93, 968

Szkody, P., Howell, S. B., Mateo, M., \& Kreidl, T. J. 1989, PASP, 101, 899

Szkody, P., Shafter, A. W., \& Cowley, A. P. 1984, ApJ, 282,236 (SSC)

Warner, B. 1995, Cataclysmic Variable Stars (Cambridge: Cambridge University Press)

Woods, J. A., Verbunt, F., Collier Cameron, A., Drew, J. E., \& Piters, A. 1992, MNRAS, 255, 237

Woudt, P. A., \& Warner, B. 2001, MNRAS, 328, 159

C. Martin Gaskell and Kam-Ching Leung: Department of Physics and Astronomy, University of Nebraska, Lincoln, NE 68588-0111, USA (kleung2@unl.edu).

Yongyun Li: Department of Astronomy, Nanjing University, Nanjing 210093, China (zyli@nju.edu.cn). 